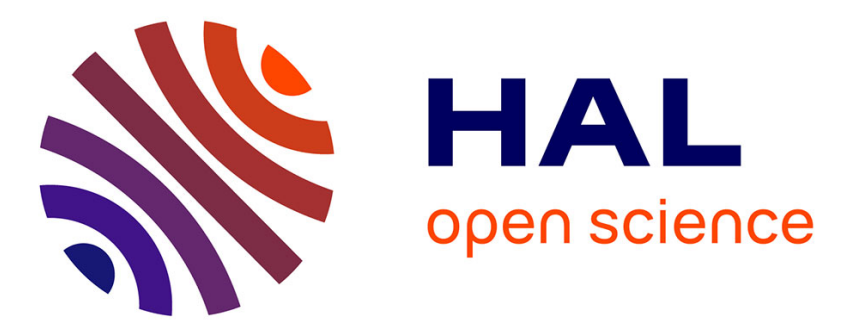

\title{
Measuring the wavenumber of guided modes in waveguides with linearly varying thickness
}

Ludovic Moreau, Jean-Gabriel Minonzio, Maryline Talmant, Pascal Laugier

\section{To cite this version:}

Ludovic Moreau, Jean-Gabriel Minonzio, Maryline Talmant, Pascal Laugier. Measuring the wavenumber of guided modes in waveguides with linearly varying thickness. Journal of the Acoustical Society of America, 2014, 135 (5), pp.2614-2624. 10.1121/1.4869691 . hal-01394317

\section{HAL Id: hal-01394317 https://hal.sorbonne-universite.fr/hal-01394317}

Submitted on 9 Nov 2016

HAL is a multi-disciplinary open access archive for the deposit and dissemination of scientific research documents, whether they are published or not. The documents may come from teaching and research institutions in France or abroad, or from public or private research centers.
L'archive ouverte pluridisciplinaire HAL, est destinée au dépôt et à la diffusion de documents scientifiques de niveau recherche, publiés ou non, émanant des établissements d'enseignement et de recherche français ou étrangers, des laboratoires publics ou privés. 
1 Measuring the wavenumber of guided modes in waveguides with

\section{linearly varying thickness}

3

4

5 Ludovic Moreau, ${ }^{\text {a) }}$ Jean-Gabriel Minonzio, ${ }^{\text {a) }}$ Maryline Talmant, ${ }^{\text {a) }}$ and Pascal Laugier ${ }^{\text {a) }}$

6

7 UPMC University Paris 06, Unité Mixte de Recherche 7623, LIP, F-75005, Paris,

8

9

10 Running title: guided modes in a thickness-varying waveguide

11

12 December $17^{\text {th }} 2013$

13 Revision February $20^{\text {th }} 2014$

14

a) Also at: CNRS, Unité Mixte de Recherche 7623, Laboratoire d'Imagerie Paramétrique, 15 rue de l'école de médecine F-75006, Paris, France. 
15

16

\section{Abstract}

Measuring guided waves in cortical bone arouses a growing interest to assess skeletal status. In most studies, a model of waveguide is proposed to assist in the interpretation of the dispersion curves. In all the reported investigations, the bone is mimicked as a waveguide with a constant thickness, which only approximates the irregular geometry of cortical bone. In this study, guided mode propagation in cortical bone-mimicking wedged plates is investigated with the aim to document the influence on measured dispersion curves of a waveguide of varying thickness and to propose a method to overcome the measurement limitations induced by such thickness variations. The singular value decomposition-based signal processing method, previously introduced for the detection of guided modes in plates of constant thickness, is adapted to the case of waveguides of slowly linearly variable thickness. The modification consists in the compensation at each frequency of the wavenumber variations induced by the local variation in thickness. The modified method, tested on bone-mimicking wedged plates, allows an enhanced and more accurate detection of the wavenumbers. Moreover, the propagation in the directions of increasing and decreasing thickness along the waveguide is investigated.

PACS numbers 43.35 Cg, 43.80.Vj, 43.20.Ye, 43.20.Mv, 43.60.Fg, 87.15.La 


\section{Introduction}

The cortical envelope of long bones has been reported to behave like a waveguide with respect to ultrasound propagation. ${ }^{1,2}$ The potential of guided waves as a diagnostic tool to assess bone status is now considered by several research groups mostly because the propagation characteristics of guided waves convey information on bone strength-relevant characteristics, such as cortical thickness and elasticity, that cannot be readily assessed by currently available X-ray imaging modalities. ${ }^{3,4}$ Moreover, compared to X-rays, ultrasound technology is also less expensive, non-ionizing and portable.

Guided mode propagation in cortical bone is investigated using the so-called axial transmission technique in which the signal propagating along the bone axis is recorded at multiple positions aligned along a same side of a skeletal site, by moving a receiver, ${ }^{5-8}$ or moving both the transmitter and the receiver in parallel, ${ }^{9}$ or using a multiple element array, ${ }^{10-}$ ${ }^{12}$, or using photo-acoustic excitation and optical detection. ${ }^{13}$ The first version of the axial transmission approach consisted in recording the time-of-flight of the earliest component of the signal recorded at the receivers, the so-called first arriving signal (FAS). ${ }^{14-18}$ In subsequent developments, a multiple frequency approach in which FAS velocity is measured at different frequencies has also been described. ${ }^{9,19,20}$

A different approach, based on a more complete analysis of the recorded time signals, consists in detecting one or more particular guided modes which are then identified by coupling the experimental analysis to a model of the waveguide. For example, Moilanen et al. have proposed to specifically detect a thickness-sensitive fundamental flexural guided wave. $^{12,21}$ Other authors analyze the full response of the waveguide using various signal processing techniques (e.g., time-frequency distributions, two-dimensional spatio-temporal Fourier transform) to measure the dispersion curves of multiple guided modes. ${ }^{6,22}$

A method combining an ultrasonic multi-element array with a singular value 
60

61

decomposition (SVD)-based signal processing has recently been proposed by our group to measure the propagation of guided waves in cortical bone. ${ }^{11,23} \mathrm{~A}$ small array has been specifically designed for in vivo measurements and to accommodate to the limited access to cortical skeletal sites, such as the distal radius at the forearm. The method, extensively described in previous publications, has been tested successfully with bone mimicking platelike waveguides ${ }^{11,23-25}$ and ex vivo human radius specimens. ${ }^{26,27}$

In most studies, a model of waveguide is proposed to assist in the interpretation of the dispersion curves. Various models have been proposed, ${ }^{2}$ including the free 2-D elastic plate, ${ }^{11,}$ 23, 28 the free 2-D gradient elastic plate ${ }^{29,30}$ or the free 3-D elastic tube. ${ }^{6,13,27,31}$ They only approximate the complex heterogeneous and geometrically irregular structure of cortical bone. In particular, in all the reported investigations, the bone is mimicked as a waveguide with a constant thickness. However, experimental observations indicate that at the distal radius, the most frequently investigated skeletal site using axial transmission, the thickness of the cortical shell varies slowly, being thinner at the proximal end (epiphysis) and thicker in the mid section (diaphysis). To the authors' best knowledge, the effect of a varying bone cortical thickness on guided modes dispersion curves has not been reported so far.

Propagation in waveguide with variable thickness has been studied theoretically, ${ }^{32-34}$ experimentally $^{35-37}$ or numerically ${ }^{36,38}$ in the context of non destructive testing, ${ }^{39}$ ocean waveguide, ${ }^{40}$ or study of musical instruments such as horns. ${ }^{34}$ In the context of ultrasonic characterization of bone, the aim of this paper is first to document the influence on measured dispersion curves of a waveguide of varying thickness and second to propose a method to overcome the measurement limitations induced by such thickness variations. A free 2-D elastic plate waveguide with slowly linearly varying thickness (typical to the configuration encountered at the distal human radius), supporting "adiabatic" propagating waves, is considered. The main advantage of such a model rather than a more realistic cortical bone 
geometry or a not-rigorously linear variation in thickness is its simplicity: it allows deriving simple analytic expressions to describe the impact on the wave numbers of a varying thickness and facilitates understanding of the effect of the varying thickness on guided mode propagation. The disadvantage, of course, is that it only approximates complex bone structure. However, 2-D elastic plate or tube models with constant thickness have previously demonstrated a high level of consistency with experimental observations in cortical bone measured ex-vivo.

The aims of this paper are twofold, (1) to gain insights into the influence of a slowly linearly varying thickness of the waveguide on guided modes, (2) to propose a method, adapted from the currently existing SVD-based signal processing, to overcome the guided mode measurement limitations induced by these thickness variations. The paper is organized as follows. A model is proposed to predict the effect on guided mode wavenumbers of a waveguide of slowly linearly varying thickness (Sec. II). The predicted variations are taken into account in the adapted signal processing technique (Sect. III). Both the model and the adapted signal processing are then validated on experimental data from bone-mimicking wedged plates (Sec. IV). Finally, the direction of propagation of the guided waves is investigated.

\section{Influence of the varying thickness on adiabatic mode wavenumbers}

\section{A. Cortical bone is considered as a plate with a linear varying thickness}

In order to illustrate the thickness variation of the cortical bone, two cross-sections images derived from 3-D X-ray computed tomography data (Siemens, Somaton 4 Plus, $200 \mu \mathrm{m}$ voxel size) of a human distal radius are shown in Fig. 1(a). These images, excerpted from Ref. 41, are illustrative of the general structure of the cortical shell of the 39 human radius specimens for which the FAS velocity was reported in Ref. 16. As illustrated on the 
111 longitudinal cross-section, the cortical thickness decreases regularly with a moderate slope

112 from the mid-diaphysis (left) to the epiphysis (distal end, right). By reanalyzing the X-ray 113 computed tomography radius database, the thickness variation along the bone axis in the 114 measurement region, highlighted with a white square on Fig. 1(a), can reasonably be 115 approximated with a linear fit. The analysis of the 39 excised human radii evidenced a mean 116 cortical thickness of $2.2 \pm 0.6 \mathrm{~mm}$ with a mean cortical angle equal to $1.2 \pm 0.7^{\circ}$.

117 The relevance of a plate versus a tube model to represent the cortical shell of human 118 radius specimens has been discussed in several studies. ${ }^{6,27,31}$ Predictions using a plate model 119 have been found to fit well the experimental data observe on ex vivo radius specimens. ${ }^{26,28}$

120 Moreover, on bone mimicking phantoms covered by a soft tissue mimicking layer, ${ }^{13,24,42,43}$ 121 indicate that the measured guided modes can be interpreted using a free plate model. Thus, an 122 elastic plate model with a linear varying thickness, although it represents a simplification compared to the complex structure of bone, is adopted here in order to evidence the effect of

124 the thickness variation on the guided mode measurement.

\section{B. Adiabatic guided modes}

The slowly varying thickness is associated with guided modes that are supposed to be adiabatic: they locally correspond to guided modes of a plate of constant thickness. If $k(e, f)$ is a valid frequency-wavenumber curve for a free elastic plate whose thickness is $e$, then the

130 frequency-wavenumber curve $k(\alpha e, f)$ for a plate of any thickness $\alpha e$ can be deduced using

131 the equation

$$
k(\alpha e, f)=1 / \alpha k(e, \alpha f),
$$

133 where $\alpha$ is a generic waveguide thickness scaling factor. The two wavenumbers given in Eq.

134 (1) correspond to identical frequency $\times$ thickness and wavenumber $\times$ thickness products. They 135 also correspond to identical phase velocity and group velocity. 
Consider two close positions $x$ and $x+d x$ along the waveguide that are associated with

137

138 thicknesses such that $e(x+d x)$ is equal to $e(x)+d e$ [Fig. 1(a)]. In order to discuss the expression given by Eq. (1), two particular plate modes A1 and S2 are illustrated on Figs. 1(b) and (c). The transition from the modes $k(x, f)$ associated with the largest thickness $e(x)$ (shown as thick lines) to the modes $k(x+d x, f)$ associated with the smallest thickness $e(x)+d e(d e$ chosen negative in this example, dashed lines) ) is manifested as a shift of the dispersion curves towards higher frequencies. An opposite effect, i.e. a shift towards lower frequencies, is observed when the thickness increases (positive de). Figure 1(c) is a zoom of a small portion of the $(f, k)$ 2-D space shown in figure 1(b) indicating the small variations $d f, d k$ and $\Delta k$ defined in Eqs. (3) and (4).

The scaling factor $\alpha$ equal to $e(x+d x) / \mathrm{e}(x)$ can be expressed as $1+d e / e(x)$. The ratio dele $(x)$ is assumed to be small compared to 1 and thus further calculus will be done with the perturbation method at the first order of the quantity dele $(x)$. Equation (1) can be written as

$$
k(x+d x, f)=k(x, f-d f)+d k .
$$

The previous equation links two wavenumbers for two different positions $x$ and $x+d x$ at two different frequencies, $f$ and $f-d f$. The small variations $d f$ and $d k$, induced by the thickness variation de, satisfy

$$
d f=-f \frac{d e}{e(x)},
$$

$$
d k=-k(x, f) \frac{d e}{e(x)}
$$

The first order Taylor expansion along frequency of wavenumber $k(x, f-d f)$ [right hand part of Eq. (2)] writes as

$$
k(x, f-d f)=k(x, f)-\frac{\partial k}{\partial f} d f .
$$

158 Using Eq. (3) and the definition of the phase and group velocities $v_{\phi}$ and $v_{g}$, the derivative 
159 term $\frac{\partial k}{\partial f} d f$ can be approximated by $\frac{v_{\phi}(x, f)}{v_{g}(x, f)} d k$. This term is shown as $\Delta k$ on Fig. 1(c).

160 Combining Eqs. (2) to (4), the difference between $k(x, f)$ and $k(x+d x, f)$, illustrated as a thick 161 arrow on Fig. 1(c), is equal to $d k-\Delta k$. Finally, the variation of the wavenumber at a fixed 162 frequency writes

$$
k(x+d x, f)=k(x, f)\left[1+\frac{d e}{e(x)}\left(\frac{v_{\phi}(x, f)}{v_{g}(x, f)}-1\right)\right] .
$$

164 Next, we introduce the term $\varepsilon(x, f)$ defined as

$$
\varepsilon(x, f)=\frac{d e}{e(x)}\left(\frac{v_{\phi}(x, f)}{v_{g}(x, f)}-1\right)
$$

166 This term is dimensionless and can be interpreted as a deviation rate measuring the

167 wavenumber variation in response to the thickness variation. Hereafter it will be referred to as 168 the "deviation term". It depends on the waveguide thickness variation rate dele $(x)$, on the guided mode being considered and on its velocity dispersion.

\section{Adiabatic condition}

The "adiabatic condition", introduced in paragraph II.A, is satisfied if the deviation

174 term $\varepsilon(x, f)$, given by Eq. (6), is small compared to 1 . This case is satisfied for moderate

175 dispersion and weak thickness variation. If mode dispersion is large, i.e. $v_{\phi}$ is large compared

176 to $v_{\mathrm{g}}, e . g$., for frequencies close to cut-off frequencies, $\varepsilon$ could be non negligible even if the

177 thickness variation dele $(x)$ is small. On the contrary, if mode dispersion is null, i.e. $v_{\mathrm{g}}$ and $v_{\phi}$

178 are equal, $\varepsilon$ is null and the thickness variation has not effect on the wavenumber. This is the

179 case for example for the Rayleigh wave which corresponds to a surface wave and is not

180 influenced by the opposite interface. Moreover, the dispersion term $v_{\phi} / v_{g}-1$ is mostly 
181 positive. It implies that $d e$ and $\varepsilon$ have the same sign: thus, an increase (respectively a 182 decrease) in thickness leads to an increase (respectively a decrease) in the wavenumber. 183 Exceptions are mode A0, for which $v_{\mathrm{g}}$ is inferior to $v_{\phi}$, and modes associated with ZGV (zero 184 group velocity) resonances, for which group and phase velocities have opposite signs. ${ }^{44}$

185 Consider that the adiabatic condition is satisfied along the propagation path of $n$ close 186 positions $x_{i}$ shown in Fig. 1(a), associated with $n$ local thicknesses $e\left(x_{i}\right)$. At a fixed frequency

$187 f$, along its propagation the wavenumber $k(x, f)$ undergoes a series of homothetic transforms 188 given by Eqs. (5) and (6). The $n^{\text {th }}$ position is linked to the first one with the following 189 relationship

$$
k\left(x_{n}, f\right)=k\left(x_{1}, f\right) \prod_{i=1}^{n-1}\left\{1+\varepsilon\left(x_{i}, f\right)\right\}
$$

with $i$ the position index ranging from 1 to $n$ [Fig 1(a)]. As all the deviation terms $\varepsilon$ are assumed to be small compared to 1, the previous equation can be approximated to the first order in $\varepsilon\left(x_{i}, f\right)$ with

$$
k\left(x_{n}, f\right)=k\left(x_{1}, f\right)\left\{1+\sum_{i=1}^{n-1} \varepsilon\left(x_{i}, f\right)\right\} .
$$

At each step, a small variation $\varepsilon\left(x_{i}, f\right) . k\left(x_{1}, f\right)$ is added to the reference wavenumber $k\left(x_{1}, f\right)$.

The measurement of the spatial variations of the wavenumber has been proposed to reconstruct the profile variation $d e(x)$ of the waveguide in case of moderate dispersion. ${ }^{45}$

\section{Wavenumber variation for a linearly varying thickness waveguide}

200 Consider a linear array with a group of receivers surrounded with two groups of transmitters. The array is in contact with a waveguide with a linearly varying thickness (Fig. 2). The 202 receivers are equally spaced, with an array pitch denoted $p$. The reference of axis $(O x)$, i.e. the 203 position $x=0$, is located at the center of the receiving array. This position is associated with the reference thickness $e_{0}$. Thus, the varying thickness $e(x)$ is given by 


$$
e(x)=e_{0}+x \cdot \tan \alpha,
$$

206 with the subscript 0 associated with values at position $x=0$. Notation "+" (respectively “_") 207 denotes the increasing (respectively decreasing) thickness direction. By convention, the first 208 receiver is placed on the thickest side, i.e., at a negative position (Fig. 2). The variation of the wavenumbers along the receivers at a fixed frequency can be obtained using Eq. (7), as long as the adiabatic condition $\varepsilon<<1$ [Eq. (6)] is satisfied at each adiabatic transform, i.e., from one receiver to the next one in this case. Moreover, the variation of the wavenumber can be expressed as

$$
k(x, f)=k\left(x_{0}, f\right)\left(1+\frac{\varepsilon\left(x_{0}, f\right)}{p} x\right)
$$

214 The term $\varepsilon\left(x_{0}, f\right)$, being the deviation term at the array center, is given by Eq. (6). In order to discuss the validity of Eq. (10), two frequencies, corresponding to two different deviation terms $\varepsilon\left(x_{0}, f\right)$, respectively, are considered in the following. at frequencies of interest in a bone-mimicking wedged plate with an angle $\alpha=2^{\circ}$ and a thickness $e_{0}=2.25 \mathrm{~mm}$. The geometrical characteristics of the wedged plate are representative of the typical values estimated from Ref. 16. The typical thickness variation $\operatorname{dele}(x)$, equal to $p \tan (\alpha) / e_{0}$, is about $1.4 \%$, with $p=0.892 \mathrm{~mm}$. Figure 3(a) shows first the dispersion curves $k\left(x_{0}, f\right)$ of this plate at $x=0$. The corresponding variations of the deviation terms $\varepsilon\left(x_{0}, f\right)$ are shown in Fig. 3(b). Two selected frequencies are marked with symbols in Figs. 3(a) and (b). The first case marked with a star (mode $\mathrm{A} 1$ at $f_{1}$ equal to $0.8 \mathrm{MHz}$ ), corresponds to a deviation term $\varepsilon\left(x_{0}, f_{1}\right)$ equal to $0.9 \%$. The second case marked with a circle (mode $\mathrm{S} 2$ at $f_{2}$ equal to 0.8 $\mathrm{MHz})$, is associated with a more pronounced deviation term $\varepsilon\left(x_{0}, f_{2}\right)$ equal to $7.7 \%$. Finally, figures 3(c) and (d) show the variation in $x$ of the wavenumbers $k\left(x, f_{1}\right)$ and $k\left(x, f_{2}\right)$ and their corresponding amplitudes $\sin \left[k\left(x, f_{1}\right) x\right]$ and $\sin \left[k\left(x, f_{2}\right) x\right]$ for the two modes at the two 
selected frequencies $f_{1}$ and $f_{2}$. The receiver positions are marked with black dots.

It can be observed that both wavenumbers $k\left(x, f_{1}\right)$ and $k\left(x, f_{2}\right)$ decrease in the direction of decreasing thickness (direction "-“). On the contrary, for the opposite direction "+", i.e., when thickness increases, the wavenumbers increase. Moreover, the variation of the wavenumbers along the axis $(O x)$ are well described by the linear approximation, given by Eq. (10) and shown with thin lines, even for the deviation $\varepsilon\left(x_{0}, f_{2}\right)=7.7 \%$. These modes with varying wavenumber can be seen as being spatially modulated, as for example classical chirps, but in the spatial domain instead of the temporal domain [Fig. 3(d)].

\section{Material and methods}

\section{A. Experimental set up}

The axial transmission setup is composed of a $1 \mathrm{MHz}$-centre frequency cMUT ultrasonic array (Vermon, Tours, France), a multi-channel array controller (Althaïs, Tours, France) and a custom made graphic interface. The cMUT array has been described in Ref. 46. Its configuration is detailed in Fig. 2: it consists of two sets of 5 transmitters on each side of a group of 24 receivers located at the centre of the array. This array with its two sets of transmitters was initially designed for soft tissues bidirectional correction. ${ }^{10,42}$ In the present work, this configuration allows waves propagation to be studied in both opposite directions, denoted "+" and "-" in Fig. 2. The array is controlled by the array controller and the graphic interface allows real-time visualization of the calculated $(f-k)$ diagrams. The pitch of the elements, denoted $p$, is equal to $0.892 \mathrm{~mm}$. The frequency bandwidth of the emitted signal, a one period burst of $1 \mathrm{MHz}$ corresponds to a $-6 \mathrm{~dB}$ power spectrum spanning the frequency range of 0.5 to $1.6 \mathrm{MHz}$. Signals are recorded at a sampling frequency of $20 \mathrm{MHz}$ and a 12-bit resolution with 16 time averages. The signals corresponding to all possible transmit-receive pairs in the array are recorded. The probe is placed in contact with the wedged plate using a 
coupling gel (Aquasonic, Parker Labs, Inc, Fairfield, NJ, USA).

The bone-mimicking plate is made of glass fibres embedded in epoxy (Sawbones Pacific Research Laboratories, Vashon, WA, USA). The following mechanical properties, ${ }^{47}$ obtained with resonant ultrasound spectroscopy, ${ }^{48}$ were used to compute the guided wave dispersion curves in the bone-mimicking plate using a 2-D transverse isotropic free plate model: $:^{24,25,49}$ mass density 1.64 g.cm ${ }^{-3}$ and stiffness coefficients (in GPa) $c_{11}=13.9$; $c_{33}=$ 20.9; $c_{55}=4.3 ; c_{13}=6.9$. The guided modes are labelled $\mathrm{A} n$ and $\mathrm{S} n$ considering their symmetry and their apparition order in frequency. In the following, measurements were performed on a bone-mimicking wedged plate, the angle of which is denoted $\alpha$. Two wedged plates with $\alpha$ equal to $1^{\circ}$ and $2^{\circ}$ were measured. A plate without angle was also measured as a reference.

\section{B. SVD-based signal processing}

The experimental setup described in section III.A allows the measurement of 2 sets of $M \times N$ temporal signals, each of which consisting of all the signals that correspond to one of the 2 directions of propagation. $M$ and $N$ are the number of receivers and transmitters, respectively. Waveguides with varying thickness have been already studied in non-destructive evaluation (NDE), where materials generally have light damping and large dimensions compared to the wavelength. Thus, large propagation paths can be recorded and analyzed using a standard post-processing technique, the so-called spatio-temporal Fourier transform, ${ }^{50}$ with the assumption that the thickness of the waveguide remains constant along the receiving aperture. $^{35,36}$ Time-frequency analysis $^{33,36}$ and analysis of the reflection coefficient ${ }^{39}$ have also been proposed. Correction in the time domain have been recently investigated to compensate the pulse dispersion caused by a varying thickness. ${ }^{51}$ In contrast to the materials investigated in NDE, cortical bone and specifically the bone mimicking material investigated here are highly damping materials. The combination of absorption and of the limited 
receiving length of the array makes that specific signal processing is therefore required to enhance the wavenumber evaluation.

A SVD-based signal processing technique was recently developed for this purpose and

283 has been extensively detailed in our previous publications. ${ }^{11,23}$ Briefly, it takes advantage of 284 the multi-transmit, multi-receive configuration of the ultrasonic array. If $s_{n m}(t)$ denotes the 285 temporal signal recorded at the receiver positioned at $x_{m}{ }^{\mathrm{R}}$ after transmission by the $n^{\text {th }}$ 286 transmitter, the main steps of the SVD-based signal processing can be summarized as follows: $s_{n m}(t)$.

2) singular value decomposition of the transfer matrix $\mathbf{S}$ at each frequency: $\mathbf{S}$ is 290 decomposed on $N$ reception singular vectors $\mathbf{U}_{n}$, and we denote by $\sigma_{n}$ the associated singular 291 value.

3) separation of signal from noise by identifying the singular values larger than a 293 heuristically determined threshold, and by keeping only the corresponding number of singular 294 vectors. This sets the rank of the matrix $\mathbf{S}$ at each frequency.

4) definition of appropriate test vectors $\mathbf{e}^{\text {test }}$ with a norm equal to 1 , expressed in the 296 receivers basis. The projection of these test vectors onto the signal subspace (i.e. the reception 297 singular vectors) leads to the so-called normalized Norm function, defined by ${ }^{11,23}$

$$
\operatorname{Norm}(f, k)=\sum_{n=1}^{r a n k}\left|\left\langle\mathbf{U}_{n} \mid \mathbf{e}^{\text {test }}\right\rangle\right|^{2}
$$

5) extraction of the guided mode wavenumbers corresponding to the maxima of the Norm function. To this end, a second threshold is heuristically defined.

These operations are performed on matrices of signals recorded with both sets of transmitters, 
diagrams, for two reasons: first, the signal is separated from noise; second, the matrix Norm is normalized, i.e. all points have their values between 0 and 1 . The maxima values do not depends on the mode energy. Maxima of the Norm function close to 1 mean that the testing vector is close to a measured mode.

However, the choice of the test vectors is critical to enhance the guide mode wavenumber measurement. In Ref. 11, the test vectors are plane waves

$$
e_{m}^{t e s t}(k)=\frac{1}{\sqrt{M}} e^{i k k_{m}^{\mathrm{R}}}
$$

where $k$ is a wavenumber corresponding to a plane wave. These test vectors are appropriate for modes propagating in a waveguide of constant thickness. Indeed, the projection of plane waves onto the basis of the singular vectors is equivalent to performing the spatial Fourier transform of the singular vectors. The method has been extended to dissipative waveguides by using a complex wavenumber. ${ }^{23}$ While this approach is appropriate for modes propagating in a waveguide of constant thickness, the developments presented in section II indicate that the approach may no longer be adapted in case of a thickness-varying waveguide.

\section{Test vector with varying wavenumber}

Paragraph II.C shows that the wavenumbers of the guided modes is affected by changes in the thickness of the waveguide. However, in the current SVD-based signal processing, the test vectors include a constant wavenumber. It may be preferable to use test vectors that fit better the physics of the problem. Towards this goal, the plane waves [Eq. (12)] are replaced by waves with a varying wavenumber following

$$
e_{m}^{t e s t}(k, \varepsilon)=\frac{1}{\sqrt{M}} e^{i k^{\text {test }}\left(x_{m}^{\mathrm{R}}\right) x_{m}^{\mathrm{R}}}
$$

with $k^{\text {test }}(x)$ defined with coefficients $k$ and $\varepsilon$ of a first order Taylor expansion following Eq. (10) as 


$$
k^{\text {test }}(x)=k\left(1+\frac{\varepsilon}{p} x\right)
$$

The example of a simple case of a single propagating mode associated with a single singular vector $\mathbf{U}_{1}$ is given to illustrate this adaptation of the signal processing. The singular vector is defined using Eqs. (10) and (14) with two arbitrary values $k_{0}$ and $\varepsilon_{0}$. In this case, the scalar product $<\mathbf{e}^{\text {test }}(k, \varepsilon) \mid \mathbf{U}_{1}>$ writes as

$$
\left\langle\mathbf{e}^{\text {test }}(k, \varepsilon) \mid \mathbf{U}_{1}\right\rangle=\frac{1}{M} \sum_{m=1}^{M} \exp \left[i\left(\left(k_{0}-k\right)+\left(k_{0} \frac{\varepsilon_{0}}{p}-k \frac{\varepsilon}{p}\right) x_{m}^{\mathrm{R}}\right) x_{m}^{\mathrm{R}}\right] .
$$

The Norm function [Eq. (11)] expresses as

$$
\operatorname{Norm}(k, \varepsilon)=\left|\left\langle\mathbf{e}^{\text {test }}(k, \varepsilon) \mid \mathbf{U}_{1}\right\rangle\right|^{2}
$$

\section{Comparison with the spatial Fourier transform and validity domain}

The two examples shown in Fig. 3 are discussed. They correspond to guided waves propagating in a bone-mimicking wedged plate with an angle $\alpha=2^{\circ}$ and a thickness $e_{0}=$ $2.25 \mathrm{~mm}$. The propagation of modes A1 and S2 was computed using Eq. (7) at a frequency of $0.8 \mathrm{MHz}$. In the first example, the propagation of mode A1 is investigated. It is represented with a star in the figures, and one can see that it corresponds to $\varepsilon_{0}=0.9 \%$ and $k_{0}=1.5 \mathrm{rad} \cdot \mathrm{mm}^{-1}$. This is considered to be a moderate wavenumber variation between the first

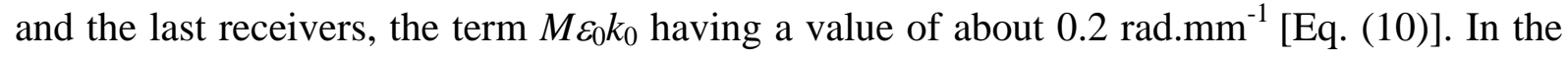
second example, we consider the propagation of mode S2, represented with a circle in the

347 figures. Although the frequency is the same as for mode $\mathrm{A} 1$, in this case $\varepsilon_{0}=7.7 \%$ and $k_{0}=$ $0.5 \mathrm{rad} . \mathrm{mm}^{-1}$. This corresponds to a larger wavenumber variation of about $1 \mathrm{rad} . \mathrm{mm}^{-1}$. The spatial Fourier transform of these two examples is shown in Figs. 4(a) and (b) with a thick

350 gray line. In the first example (mode A1), the peak is located at $k=k_{0}$, and thus the 351 corresponding moderate wavenumber variation does not affect the ability of the spatial 
352 Fourier transform to evaluate the wavenumber at the center of the array. On the other hand, in

353 the second example (mode S2), the spatial Fourier transform exhibits two maxima that are

354 shifted compared to the pick value located at $k_{0}$. Thus, the larger wavenumber variation 355 prevents the evaluation of an accurate estimate of the wavenumber. In the single mode case 356 discussed here, the spatial Fourier transform corresponds to the plane wave test vectors 357 [Eq. (12)] as described in Ref. 11. It is then calculated with $\varepsilon=0$ in Eq. (16) and therefore it is 358 indicated as $\operatorname{Norm}(k, 0)$ in Figs. 4(a) and (b).

In order to illustrate the signal processing using a modified test vector [Eqs. (13) to

360 (16)], the Norm function is shown in the $(k, \varepsilon)$ plane in Figs. 4(c) and (d). The Norm function 361 presents a single peak centered at the point $\left(k_{0}, \varepsilon_{0}\right)$, shown with a star (A1) and a circle (S2).

362 In order to compare with the spatial Fourier transform, the line corresponding to $\varepsilon=\varepsilon_{0}$ is 363 shown as a thin black line, and is indicated as $\operatorname{Norm}\left(k, \varepsilon_{0}\right)$ in Figs. 4(a) and (b). It can be 364 observed that the value of the maxima is 1 . A maximum value close to 1 suggests that the 365 correction proposed in Eq. (14), using the modified test vector, has succeeded to compensate 366 for the wavenumber variation due to the varying thickness.

The measurement limit domains are indicated with thick lines. The lowest measurable wavenumber, $\pi / L$, corresponds to a wavelength equal to half the extent of the receiving area equal to $L$ or $M p$. The highest measurable wavenumber, $2 \pi / p$, corresponds to the sampling wavenumber, denoted $k_{s}$. In this case, the wavelength is equal to the array pitch $p$. Indeed, as

371 the guided modes are recorded in only one propagation direction, the measured wavenumber 372 have only one (positive) sign and thus the Nysquist limit ( $k$ inferior to $\left.k_{s} / 2\right)$ can be exceeded 373 until $k$ equal to $k_{\mathrm{s}}$. The line $\varepsilon=10 \%$ corresponds to the upper limit of the adiabatic condition 374 discussed in paragraph II.C. Thus below $10 \%$, the deviation term $\varepsilon(x, f)$ given by Eq. (6) is 375 considered small compared to 1 , and the linear variation of the wavenumber [Eq. (10)] is 376 valid. 
The resolution is given by the mid peak values. Resolutions in $k$ and $\varepsilon$, denoted $\delta k$ and $\delta \varepsilon$, respectively, are equal to

$$
\begin{aligned}
& \delta k=2 \pi / L, \\
& \delta \varepsilon=4 \pi /\left(M k_{0} L\right),
\end{aligned}
$$

These values are illustrated in Fig. 4 with horizontal and vertical thin arrows around the peaks. The resolution $\delta k$ is the same as in the case of the plane wave test vectors and depends only on the receiving length $L .{ }^{11}$ In addition to $L$, the resolution $\delta \varepsilon$ depends on the wavenumber $k_{0}$ and the number of receivers $M$. The domain of validity is divided into two zones, denoted I and II. If the couple $\left(\varepsilon_{0}, k_{0}\right)$ is located in zone I, the associated peak intercepts the $\varepsilon=0$ line. Thus following Eq. (18), the limit between the two zones corresponds to $\varepsilon k$ less than $4 \pi /(M L)$. Thus in zone I, is it possible to localize the position of the maxima (i.e., $k=k_{0}$ ) using the spatial Fourier Transform as illustrated with the A1 mode. The peaks given by the two methods are located at the same wavenumbers equal to $k_{0}$, but the peak maximum given by the spatial Fourier transform is lower than the value given by the proposed method (about 0.8 instead of 1$)$. On the contrary, if the couple $\left(\varepsilon_{0}, k_{0}\right)$ is located in zone II, i.e., $\varepsilon_{0} k_{0}$ larger than $4 \pi /(M L)$, then the peak does not intercept the $\varepsilon=0$ line and therefore it is not possible to localize the maxima using the spatial Fourier Transform as illustrated with the S2 mode. However, the correction proposed in Eq. (14), allows the detection of the modes, even in zone II as long as the deviation term is less than $10 \%$, and the function $\operatorname{Norm}(k, \varepsilon)$ exhibits a unique peak associated with a maximum value close to 1 and located at $\left(k_{0}, \varepsilon_{0}\right)$.

In conclusion, these examples illustrate that the proposed approach where the plane wave vector has been changed to a test vector with a varying wavenumber leads to a better mode detection, and consequently to a more accurate wavenumber measurement. 


\section{Results and discussion}

403 In this section, experimental data are collected on three wedged plates made of bone404 mimicking material. The thickness $e_{0}$ of the plates at the center of the receiving area is equal 405 to $2.25 \mathrm{~mm}$. The wedge angles $\alpha$ are equal to 0,1 and $2^{\circ}$. All calculations have been done 406 keeping the five singular vectors [i.e., the rank is equal to 5 in Eq. (11)] and with a second 407 threshold equal to 0.6. Whereas the Norm function computed with a plane wave test vector 408 depends on two parameters $(f, k)$ using the plane wave test vector [Eq. (12)], the new Norm 409 function computed with a test vector with varying wavenumber [Eqs. (13) and (14)] depends 410 on the three parameters $f, k$ and $\varepsilon$. The results are represented in Fig. 5 for the bone411 mimicking wedged plates with a wedge angle $\alpha=1^{\circ}$ [Fig. 5(b) and (c)] and $\alpha=2^{\circ}$ [Fig. 5(d)

412 and (e)]. Results calculated with the modified test vector $k^{\text {test }}(x)$ (circles) are compared with 413 those obtained with the plane wave test vector (dots) for both directions "+" and "-". The case 414 of the plate of constant thickness $\left(\alpha=0^{\circ}\right)$, is shown in Fig. 5(a) for reference. The main 415 observations are as follows:

416 1. Incomplete portions of branches of guided modes are detected in the wedged plates 417 compared to the plate of constant thickness.

418 2. This effect is more pronounced in the direction "+" compared to the opposite direction.

3. The length of the detected branches with the plane wave test vector decreases when the angle increases.

4. Several branches of guided modes in the wedged plates that are incompletely detected with the plane test vector (e.g., S0, A3 plate with $\alpha=1^{\circ}$, direction “_-“; S0, A1, S1, A3 plate with $\alpha=1^{\circ}$, direction "-_") can be detected using the modified test vector. Guided mode branches measured only with the modified method are indicated with thin arrows. It corresponds to wavenumbers located in zone II of Fig. 4. 
5. Some modes are even not detected at all (e.g., A3; directions "+") with either the plane wave or modified test vector. Guided mode branches not measured with any of the two methods are marked with thick black arrows. case [Fig. 5(a)]. The two first modes A0 and S0 do not have cut-off frequencies unlike the three following modes A1 (0.4 MHz), S2 (0.7 MHz) and A3 (1.3 MHz). Modes A2 and S3 are not measured. Let us consider first the results obtained with the plane wave test vectors (dots). Direction "+" (right panels) is more severely affected compared to direction "-". At $1^{\circ}$, the mode A3 is no longer detected. For modes A1 and S2, low wavenumbers with values below 0.5 rad. $\mathrm{mm}^{-1}$, are missing. The mode A1 also disappears at high wavenumbers, with values above 2 rad. $\mathrm{mm}^{-1}$. For the $2^{\circ}$ wedged plate, in addition to the mode A3, the mode S2 also completely disappears. The mode A1 is not measured for wavenumbers below $1 \mathrm{rad} . \mathrm{mm}^{-1}$. 440 For direction “-” (left panels), similar but less important alterations of the branches can be observed. For example, at $1^{\circ}$, A3 is partially detected while A1 and S2 seem to be correctly detected. At $2^{\circ}$, A3 is no longer detected and S2 is partially detected. For high wavenumbers, modes S0 and A1 are not detected. Mode A0 is the only mode that does not seem to be affected for both directions.

Some branches of guided modes that are not detected with the plane test vector can be measured using the modified test vector $k^{\text {test }}(x)$ [Eq. (14)]. These branches are indicated with thin arrows. This effect is particularly visible for the $2^{\circ}$ wedged plate and direction "-" [Fig. 448 5(d)] on modes S0, A1, S2, A3. Almost all branches lost using the plane test vector can be recovered. Similar effect is observed for the $2^{\circ}$ wedged plate and direction "+" [Fig. 5(e)]. Note that mode A3 cannot be measured in both wedged plates with any of the two methods. 
452

453

effect of the varying thickness increases with the wedge angle and with mode dispersion. Remember that the mode dispersion is high close to cut off frequencies, particularly for modes S2 and A3. The effect also increases with the wavenumber values as observed for modes S0 and A1. Secondly, the observation of direction "+" being more affected than direction "-" can be interpreted by the fact that, the plate being too thin under the transmitters, some modes such as S2 and A3 cannot be excited and subsequently cannot be measured by the receivers. On the contrary, for direction "-", these modes are excited under the transmitters and can propagate and can be measured. However these modes may vanish before the end of the receiving length, as for example S2 at $0.8 \mathrm{MHz}$ and $x$ about $10 \mathrm{~mm}$ [Fig. 3(c)]. This is similar to the phenomenon described as "acoustic black holes" for the flexural waves propagating in wedges with thickness decreasing with a power law exponent larger than $2 .^{52}$ Moreover in our case, as the bone-mimicking material is absorbing, no reflections are observed. ${ }^{53}$

Using the modified test vector allows the estimation not only of the wavenumbers as discussed above, but also of the deviation term $\varepsilon$ as shown in Fig. 4. The theoretical value of $\varepsilon\left(x_{0}, f\right)$ is plotted versus frequency for modes A1, S1 and A3 in Fig. 6 for both angles $\alpha=1$ and $\alpha=2^{\circ}$. Experimental values (shown with symbols), measured in direction “_“, are compared to the theoretical ones, showing good agreement for both plates. The experimental deviation term $\varepsilon$ is slightly underestimated. Moreover, close to cut off frequencies, the detection of the maxima can become unstable because the dispersion term $v_{\phi} / v_{g}-1$ tends towards infinity. This suggests that the limit to the linear approximation for the variations of the wavenumbers is $\varepsilon$ of the order of $10 \%$ (Fig. 4). Above this value, the test vectors defined in Eq. (14) are not adapted anymore and another type of variation should be considered (e.g. polynomial approximation of higher order). Attenuation could be also taken into account as the imaginary part of the wavenumber also varies spatially. 
Given the relatively low signal-to-noise ratio in axial transmission measurements of cortical bone, we believe that the improvement in the detection of branches brought by the modified version of the test vector may represent a significant progress. Moreover, previous results suggest that direction "-" is potentially better for cortical bone characterization. As a consequence, further assessment of the method on ex vivo specimens as well as in in vivo measurement conditions is warranted.

\section{Conclusion}

This paper introduces a modified signal processing approach adapted to the measurement of guided wave propagation in waveguides of variable thickness. The method is based on an equation that describes the evolution of the guided modes wavenumbers with respect to position along the direction of propagation in the wedged plate. Both the equation and the signal processing were validated using experimental data recorded with bone-mimicking wedged plates. This new approach to detect guided waves in wedged plates exhibits enhanced sensitivity and accuracy compared to the previous one that does not account for the thickness-

492 related variations of the wavenumbers. Indeed, typical angles of approximately $1^{\circ}$ to $2^{\circ}$ 493 observed in the cortical layer of the radius affect the propagation of the guided waves and 494 prevent large parts of the guided mode branches to be detected with the current signal 495 processing. The modified signal processing has therefore a better potential for investigation of 496 the inverse problem aiming at retrieving estimates of thickness and elastic properties of the 497 cortical bone waveguide.

\section{Acknowledgments}


Moreau et al.

JASA

501 Authors would like to acknowledge Pascal Dargent for his help for preparing the wedged 502 plates.

503 Jean-Gabriel Minonzio would like to dedicate the paper to the memory of Franck David 504 Philippe who passed away during the preparation of the manuscript.

505

506 
508 1. P. Moilanen, "Ultrasonic guided waves in bone," IEEE Trans. Ultrason. Ferroelectr. $509 \quad$ Freq. Control 55, 1277-1286 (2008).

510 2. M. Talmant, J. Foiret, and J. G. Minonzio, Bone Quantitative ultrasound Chap. 7 $511 \quad$ Guided waves in cortical bones (Springer, New York, 2010).

512 3. R. M. D. Zebaze, A. Ghasem-Zadeh, A. Bohte, S. Iuliano-Burns, M. Mirams, R. I. 513 Price, E. J. Mackie, and E. Seeman, "Intracortical remodelling and porosity in the distal radius and post-mortem femurs of women: a cross-sectional study," Lancet $\mathbf{3 7 5}$,

5. P. Moilanen, P. H. F. Nicholson, T. Karkkainen, Q. Wang, J. Timonen, and S. Cheng, 1729-1736 (2010).

4. P. Laugier, "Instrumentation for in vivo ultrasonic characterization of bone strength,"

6. D. Ta, W. Q. Wang, Y. Y. Wang, L. H. Le, and Y. Q. Zhou, "Measurement of the Dispersion and Attenuation of Cylindrical Ultrasonic Guided Waves in Long Bone," Ultrasound Med. Biol. 35, 641-652 (2009).

7. K. L. Xu, D. Ta, P. Moilanen, and W. Q. Wang, "Mode separation of Lamb waves based on dispersion compensation method," J. Acoust. Soc. Am. 131, 2714-2722 (2012).

527 8. X. J. Song, D. A. Ta, and W. Q. Wang, "A Base-Sequence-Modulated Golay Code Improves the Excitation and Measurement of Ultrasonic Guided Waves in Long Bones," IEEE Trans. Ultrason. Ferroelectr. Freq. Control 59, 2580-2583 (2012).

9. A. Tatarinov, V. Egorov, N. Sarvazyan, and A. Sarvazyan, "Multi-frequency axial 

http://dx.doi.org/10.1016/j.ultras.2013.09.025 (date last viewed 02/20/14).

10. E. Bossy, M. Talmant, M. Defontaine, F. Patat, and P. Laugier, "Bidirectional axial transmission can improve accuracy and precision of ultrasonic velocity measurement in cortical bone: A validation on test materials," IEEE Trans. Ultrason. Ferroelectr. Freq. Control 51, 71-79 (2004).

11. J. G. Minonzio, M. Talmant, and P. Laugier, "Guided wave phase velocity measurement using multi-emitter and multi-receiver arrays in the axial transmission configuration," J. Acoust. Soc. Am. 127, 2913-2919 (2010).

12. P. Moilanen, M. Maatta, V. Kilappa, L. Xu, P. H. F. Nicholson, M. Alen, J. Timonen, T. Jamsa, and S. Cheng, "Discrimination of fractures by low-frequency axial transmission ultrasound in postmenopausal females," Ost. Int. 24, 723-730 (2013).

13. P. Moilanen, Z. Zhao, P. Karppinen, T. Karppinen, V. Kilappa, J. Pirhonen, R. Myllylä, E. Hæggström, and J. Timonen, "Photo-acoustic Excitation and Optical Detection of Fundamental Flexural Guided Wave in Coated Bone Phantoms," Ultrasound Med. Biol. 40, 521-531 (2014).

14. G. Lowet and G. VanderPerre, "Ultrasound velocity measurement in long bones: Measurement method and simulation of ultrasound wave propagation," J. Biomechanics 29, 1255-1262 (1996).

15. M. Daugschies, K. Rohde, C.-C. Glüer, and R. Barkmann, "The preliminary evaluation of a $1 \mathrm{MHz}$ ultrasound probe for measuring the elastic anisotropy of human cortical bone," Ultrasonics 54, 4-10 (2014).

16. E. Bossy, M. Talmant, F. Peyrin, L. Akrout, P. Cloetens, and P. Laugier, "An in vitro study of the ultrasonic axial transmission technique at the radius: $1-\mathrm{MHz}$ velocity measurements are sensitive to both mineralization and intracortical porosity," J. Bone Mineral Res. 19, 1548-1556 (2004). 
557 17. J. Foiret, Q. Grimal, M. Talmant, R. Longo, and P. Laugier, "Probing heterogeneity of cortical bone with ultrasound axial transmission," IEEE Trans. Ultrason. Ferroelectr. Freq. Control 60, 187-193 (2013).

18. K. I. Lee and S. W. Yoon, "Correlations between ultrasonic guided wave velocities 561 and bone properties in bovine tibia in vitro," J. Acoust. Soc. Am. 131, EL375-EL381 (2012).

19. A. Sarvazyan, A. Tatarinov, V. Egorov, S. Airapetian, V. Kurtenok, and C. J. Gatt, "Application of the dual-frequency ultrasonometer for osteoporosis detection," Ultrasonics 49, 331-337 (2009).

20. V. Egorov, A. Tatarinov, N. Sarvazyan, R. Wood, L. Magidenko, S. Amin, S. Khosla, R. J. Ruh, J. M. Ruh, and A. Sarvazyan, "Osteoporosis detection in postmenopausal women using axial transmission multi-frequency bone ultrasonometer: Clinical findings," Ultrasonics, http://dx.doi.org/10.1016/j.ultras.2013.08.017 (date last viewed 02/20/14).

21. V. Kilappa, K. Xu, P. Moilanen, E. Heikkola, D. Ta, and J. Timonen, "Assessment of the Fundamental Flexural Guided Wave in Cortical Bone by an Ultrasonic AxialTransmission Array Transducer," Ultrasound Med. Biol. 39, 1223-1232 (2013).

22. V. C. Protopappas, D. I. Fotiadis, and K. N. Malizos, "Guided ultrasound wave propagation in intact and healing long bones," Ultrasound Med. Biol. 32, 693-708 (2006).

23. J. G. Minonzio, J. Foiret, M. Talmant, and P. Laugier, "Impact of attenuation on guided mode wavenumber measurement in axial transmission on bone mimicking plates," J. Acoust. Soc. Am. 130, 3574-3582 (2011).

24. J. A. Chen, J. Foiret, J. G. Minonzio, M. Talmant, Z. Q. Su, L. Cheng, and P. Laugier, 

using ultrasonic axial transmission," Phys. Med. Biol. 57, 3025-3037 (2012).

25. J. A. Chen, J. Foiret, J. G. Minonzio, Z. Q. Su, L. Cheng, and P. Laugier, "Corrigendum: Measurement of guided mode wavenumbers in soft tissueâ€"bone mimicking phantoms using ultrasonic axial transmission," Phys. Med. Biol. 58, 85938595 (2013).

26. J. Foiret, J. Minonzio, M. Talmant, and P. Laugier, "Cortical bone quality assessment using quantitative ultrasound on long bones," Engineering in Medicine and Biology Society (EMBC), Annual International Conference of the IEEE, 1121-1124 (2012).

27. J. Foiret, J.-G. Minonzio, P. Laugier, and M. Talmant, "Determination of bone properties from Lamb type of waves," POMA 19, 075091 (2013).

28. P. Moilanen, P. H. F. Nicholson, V. Kilappa, S. Cheng, and J. Timonen, "Measuring guided waves in long bones: Modeling and experiments in free and immersed plates," Ultrasound Med. Biol. 32, 709-719 (2006).

29. M. G. Vavva, V. C. Protopappas, L. N. Gergidis, A. Charalambopoulos, D. I. Fotiadis, and D. Polyzos, "Velocity dispersion of guided waves propagating in a free gradient elastic plate: Application to cortical bone," J. Acoust. Soc. Am. 125, 3414-3427 (2009).

30. A. Papacharalampopoulos, M. G. Vavva, V. C. Protopappas, D. I. Fotiadis, and D. Polyzos, "A numerical study on the propagation of Rayleigh and guided waves in cortical bone according to Mindlin's Form II gradient elastic theory," J. Acoust. Soc.

31. P. Moilanen, P. H. F. Nicholson, V. Kilappa, S. L. Cheng, and J. Timonen, "Assessment of the cortical bone thickness using ultrasonic guided waves: Modelling and in vitro study," Ultrasound Med. Biol. 33, 254-262 (2007).

606 32. V. Pagneux and A. Maurel, "Lamb wave propagation in elastic waveguides with 
607

608

609

610

611

612

613

614

615

616

617

618

619

620

621

622

623

624

625

626

627

628

629

630

631

variable thickness," Proceedings of the Royal Society A: Mathematical, Physical and Engineering Science 462(2068), 1315-1339 (2006).

33. L. De Marchi, A. Marzani, N. Speciale, and E. Viola, "Prediction of pulse dispersion in tapered waveguides," NDT \& E International 43, 265-271 (2010).

34. N. Amir, V. Pagneux, and J. Kergomard, "A study of wave propagation in varying cross-section waveguides by modal decomposition .2. Results," J. Acoust. Soc. Am. 101, 2504-2517 (1997).

35. M. E. C. El-Kettani, F. Luppe, and A. Guillet, "Guided waves in a plate with linearly varying thickness: experimental and numerical results," Ultrasonics 42, 807-812 (2004).

36. P. Marical, M. E. C. El-Kettani, and M. V. Predoi, "Guided waves in elastic plates with Gaussian section variation: Experimental and numerical results," Ultrasonics 47, 1-9 (2007).

37. Z. Hamitouche, M. E. C. El-Kettani, J. L. Izbicki, and H. Djelouah, "Reflection at the Cut-off and Transmission by Tunnel Effect in a Waveguide with Linear Section Variation," Acta Acustica United with Acustica 95, 789-794 (2009).

38. J. B. Doc, S. Felix, and B. Lihoreau, "Coarse-grid computation of the one-way propagation of coupled modes in a varying cross-section waveguide," J. Acoust. Soc. Am. 133, 2528-2532 (2013).

39. R. Carandente, J. Ma, and P. Cawley, "The scattering of the fundamental torsional mode from axi-symmetric defects with varying depth profile in pipes," J. Acoust. Soc. Am. 127, 3440-3448 (2010).

40. J. M. Arnold and L. B. Felsen, "Intrinsic Modes in a Nonseparable Ocean Waveguide," J. Acoust. Soc. Am. 76, 850-860 (1984).

41. E. Bossy, M. Talmant, and P. Laugier, "Three-dimensional simulations of ultrasonic 

axial transmission velocity measurement on cortical bone models," J. Acoust. Soc. Am. 115, 2314-2324 (2004).

634 42. L. Moreau, J. G. Minonzio, J. Foiret, E. Bossy, M. Talmant, and P. Laugier, "Accurate measurement of guided modes in a plate using a bidirectional approach," J. Acoust. Soc. Am. 135, EL15-EL21 (2014).

43. T. Tran, L. Stieglitz, Y. J. Gu, and L. H. Le, "Analysis of Ultrasonic Waves Propagating in a Bone Plate over Awater Half-Space with and without Overlying Soft Tissue," Ultrasound Med. Biol. 39, 2422-2430 (2013).

44. C. Prada, D. Clorennec, and D. Royer, "Local vibration of an elastic plate and zerogroup velocity Lamb modes," J. Acoust. Soc. Am. 124, 203-212 (2008).

45. M. E. C. El Kettani, P. Marical, and Z. Hamitouche, "Inverse Problem for the Geometry Profile Determination of Waveguides With Varying Section Using Adiabatic Behavior of Guided Waves," IEEE Trans. Ultrason. Ferroelectr. Freq. Control 56, 2023-2026 (2009).

46. D. Certon, R. Ternifi, A. Boulme, M. Legros, J. G. Minonzio, M. Talmant, F. Patat, and J. P. Remenieras, "Low frequency cMUT technology: Application to measurement of brain movement and assessment of bone quality," IRBM 34, 159-166 (2013).

47. S. Bernard, Q. Grimal, S. Haupert, and P. Laugier, "Assessment of anisotropic elasticity of small bone samples with resonant ultrasound spectroscopy: Attenuation does not prevent the measurements," Ultrasonics Symposium (IUS), IEEE International, 1599-1602, (2011).

48. S. Bernard, Q. Grimal, and P. Laugier, "Accurate measurement of cortical bone elasticity tensor with resonant ultrasound spectroscopy," Journal of the Mechanical Behavior of Biomedical Materials 18, 12-19 (2013). 
657 49. S. H. Rhee, J. K. Lee, and J. J. Lee, "The group velocity variation of Lamb wave in

658

659

660

661

662

663

664

665

666

667

668

669

670

671

672 fiber reinforced composite plate," Ultrasonics 47, 55-63 (2007).

50. D. Alleyne and P. Cawley, "A 2-Dimensional Fourier-Transform Method for the Measurement of Propagating Multimode Signals," J. Acoust. Soc. Am. 89, 1159-1168 (1991).

51. L. De Marchi, A. Marzani, and M. Miniaci, "A dispersion compensation procedure to extend pulse-echo defects location to irregular waveguides," NDT \& E International 54, 115-122 (2013).

52. V. V. Krylov and F. J. B. S. Tilman, "Acoustic 'black holes' for flexural waves as effective vibration dampers," Journal of Sound and Vibration 274, 605-619 (2004).

53. V. V. Krylov and R. Winward, "Experimental investigation of the acoustic black hole effect for flexural waves in tapered plates," Journal of Sound and Vibration 300, 4349 (2007). 
673 Figure 1. X-ray computed tomography cross sections of a human distal radius (Siemens, 674 Somaton 4 Plus) excerpted from Ref. 41, illustrating cortical bone thickness variations in a 675 typical ultrasound measurement region (a), wavenumber $k$ vs frequency $f$ for two particular 676 guided modes A1 and S2 (b) associated with the thickness $e$ at position $x$ (thick lines) and $677 e+d e$ at position $x+d x$ (thin lines), panel (c) is a zoom of (b) showing the small variations $d f$, $678 d k$ and $\Delta k$ used in Eqs. (3) to (6).

679

680 Figure 2. Configuration of the wedged plate with the elements of the ultrasonic array.

682 Figure 3. Wavenumbers $k\left(x_{0}, f\right)$ of modes A1 and S2 vs frequency in a $2.25 \mathrm{~mm}$-thick bone 683 mimicking plate (a), corresponding deviation terms $\varepsilon\left(x_{0}, f\right)$ [Eq. (6)] for $\alpha=2^{\circ}$ (b),

684 685

wavenumbers $k\left(x, f_{1}\right)$ and $k\left(x, f_{2}\right)$ (c) and associated spatial variations $\sin \left[k\left(x, f_{1},\right) x\right]$ and $\sin \left[k\left(x, f_{2}\right) x\right]$ (d) of the two modes with respect to the propagation distance $x$ at two particular frequencies shown with symbols for $\mathrm{A} 1$ at $f_{1}=0.8 \mathrm{MHz}$ (star) and S2 at $f_{2}=0.8 \mathrm{MHz}$ (circle) in (a) and (b) (points indicate the position of the receivers of the array).

Figure 4. Norm functions given by Eq. (16) in the $(k, \varepsilon) 2$-D space in a case of a single mode given by Eq. (10) for the two examples shown in Fig. 3: mode $\mathrm{S} 2$ at $0.8 \mathrm{MHz}$ with $\varepsilon_{0}$ equal to $7.7 \%$ and $k_{0}$ equal to $0.5 \mathrm{rad} . \mathrm{mm}^{-1}$ (a) and (c) and mode $\mathrm{A} 1$ at $0.8 \mathrm{MHz}$ with $\varepsilon_{0}$ equal to $0.9 \%$ and $k_{0}$ equal to 1.5 rad. $\mathrm{mm}^{-1}$ (b) and (d). The Norm functions are also represented for $\varepsilon=0$ (thick gray lines) and $\varepsilon=\varepsilon_{0}$ (thin black lines) in (a) and (b). The case $\varepsilon=0$ corresponds to the previous signal processing using plane wave test vectors [Eq. (12)] and is equivalent to the spatial Fourier transform in the single mode case. The resolution values in the $(k-\varepsilon) 2-\mathrm{D}$ space given by Eqs. (17) and (18) are shown with thin arrows. The thick lines corresponds to validity domains of zone I (spatial Fourier transform) and zone II (modified test vector). 
700 Figure 5. (color online) Experimental wavenumbers obtained with the plane wave test vector

701 for a plate of constant thickness $e=2.25 \mathrm{~mm}$ (a). Experimental wavenumbers obtained with

702 the plane wave test vector (dots) and the modified test vectors $k^{\text {test }}(x)$ (circles) on wedged 703 bone mimicking plates with central thickness $e_{0}$ equal to $2.25 \mathrm{~mm}$ and $\alpha$ equal to $1^{\circ}$ (b) and

704 (c) and $2^{\circ}$ (d) and (e). Experimental wavenumbers are compared with the theoretical modes of 705 the free plate of constant thickness $e_{0}$ (continuous and dashed lines). Results of the 706 propagation in the decreasing thickness direction (direction "-") are shown in (b) and (d).

707 Results of the propagation in the increasing thickness direction (direction "+") are shown in

708 (c) and (e). Thick arrows indicate portions of branches of guided mode not measured with any 709 of the two methods. Thin arrows indicate portions of branches of guided modes measured 710 only with the modified method. It corresponds to wavenumbers located in zone II of Fig. 4. 711 The two examples shown in Figs. 3 an 4 are reported in (d) with the same symbols (large 712 circle and star).

713

714 Figure 6. Theoretical (continuous and dashed lines) and experimental values of the deviation 715 term $\varepsilon\left(x_{0}, f\right)$ for $\alpha=1^{\circ}$ (a) and $\alpha=2^{\circ}$ (b), for modes A1 (circles), S2 (stars) and A3 (dots) for 716 direction “-”. 


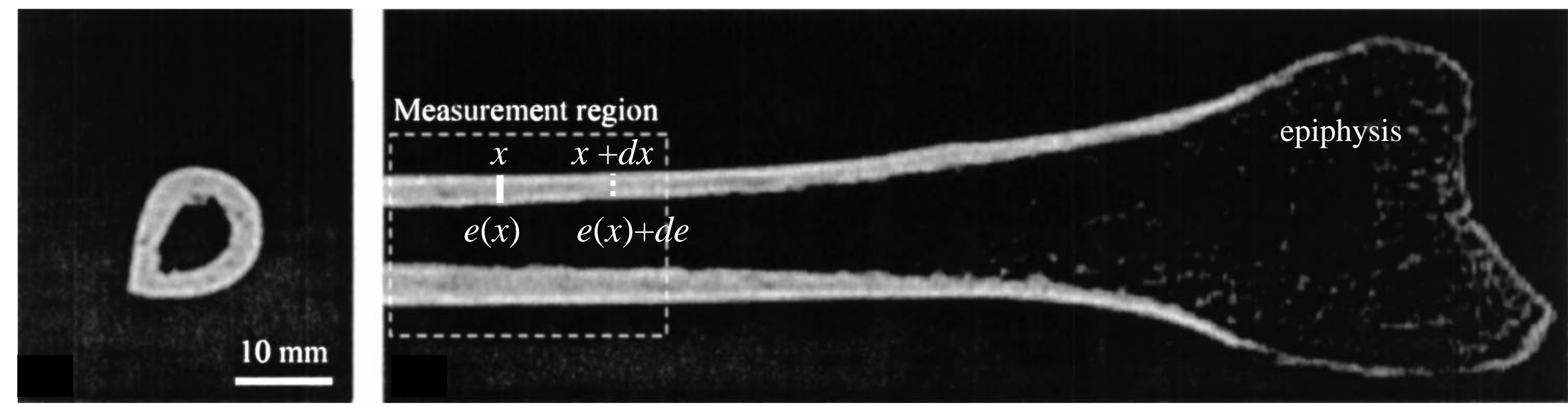

(a)
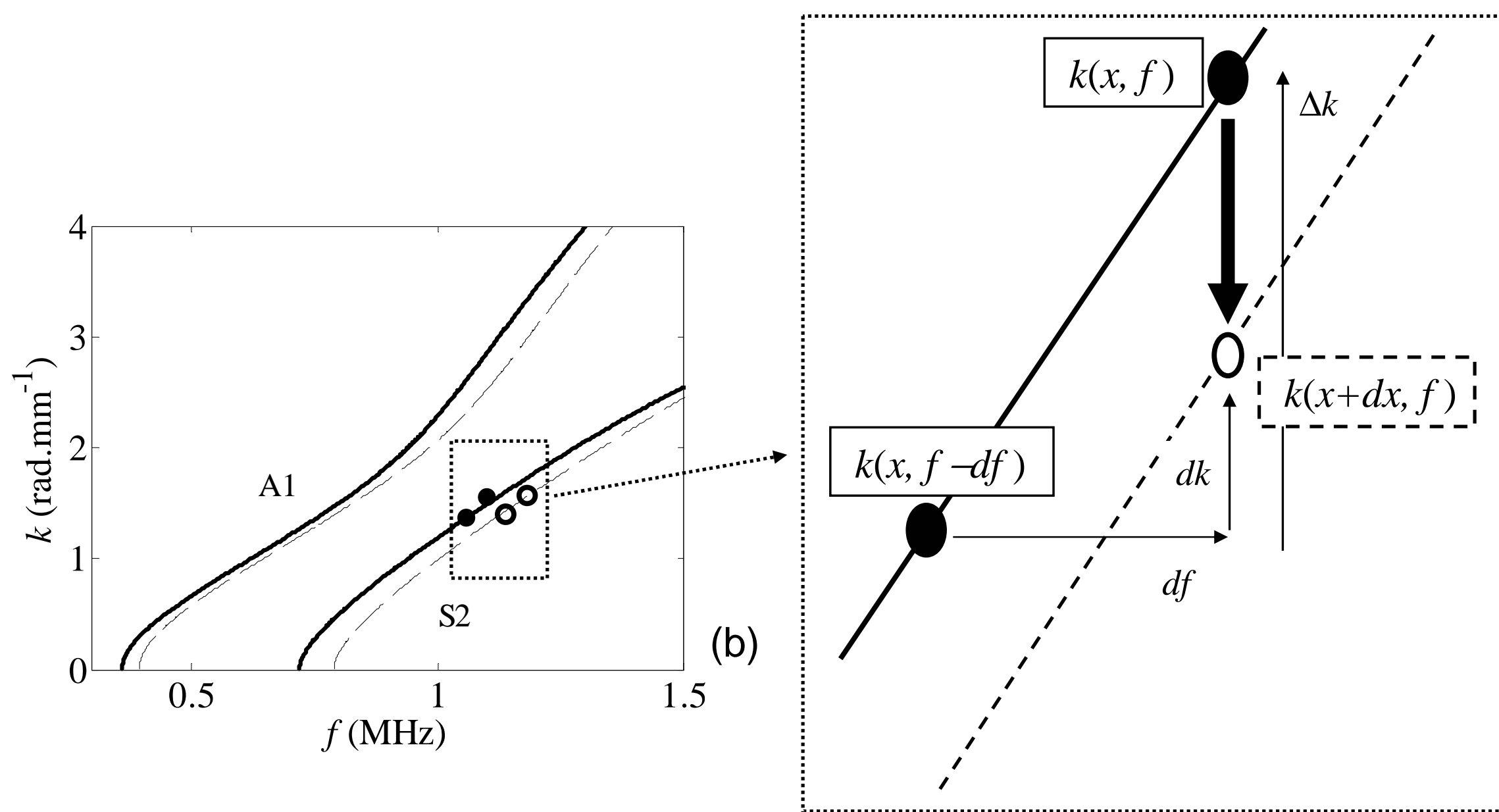


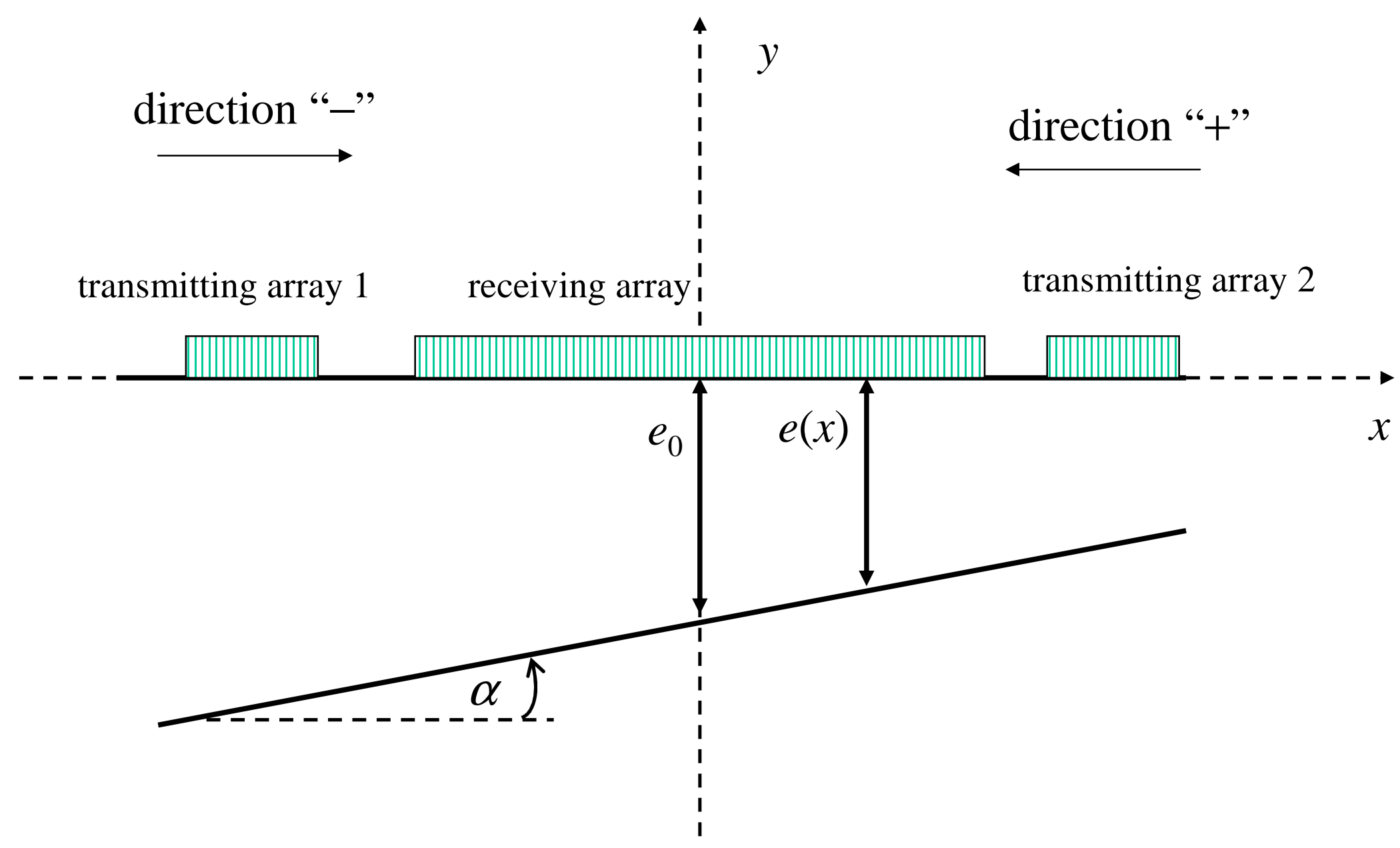



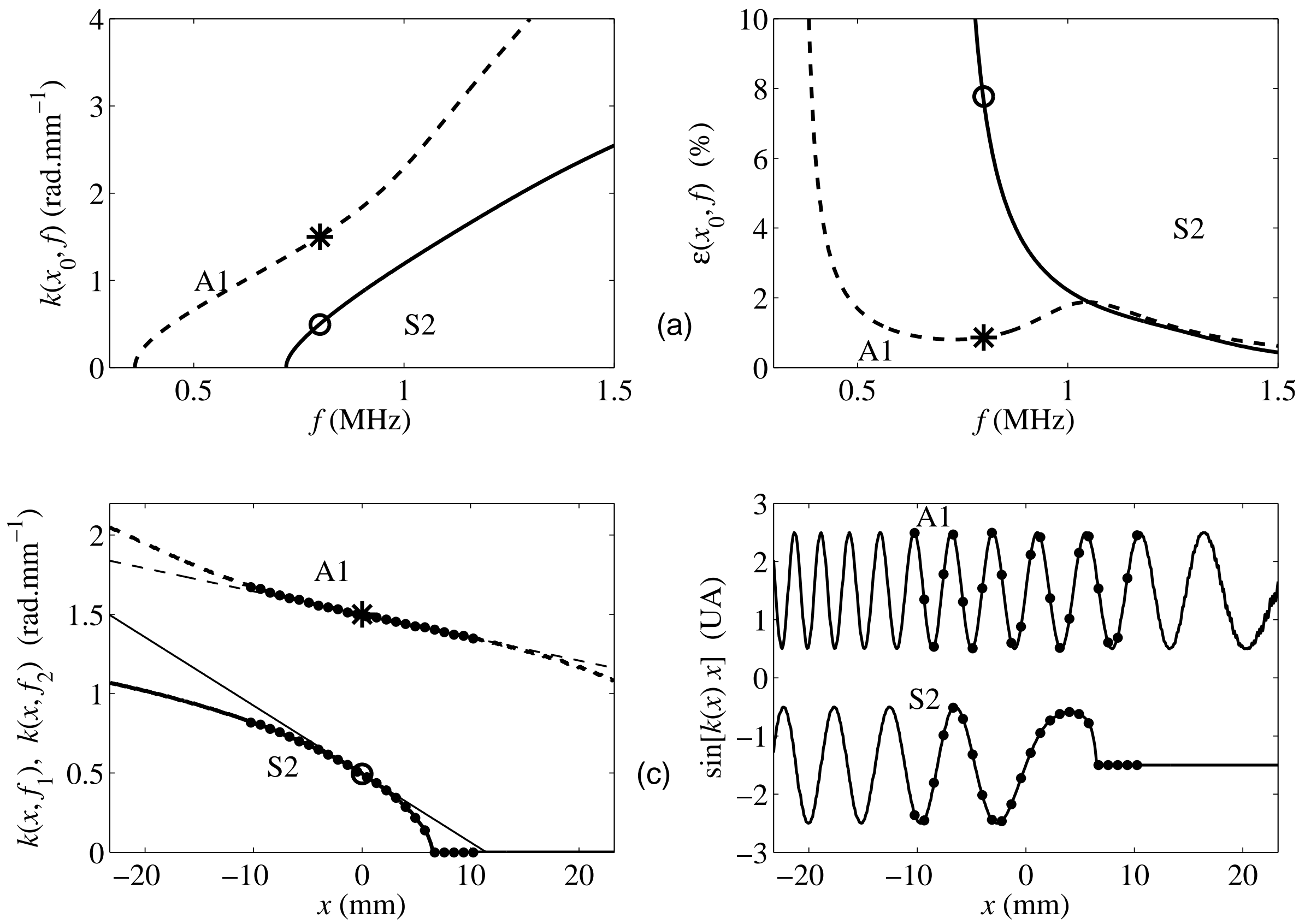

(d) 

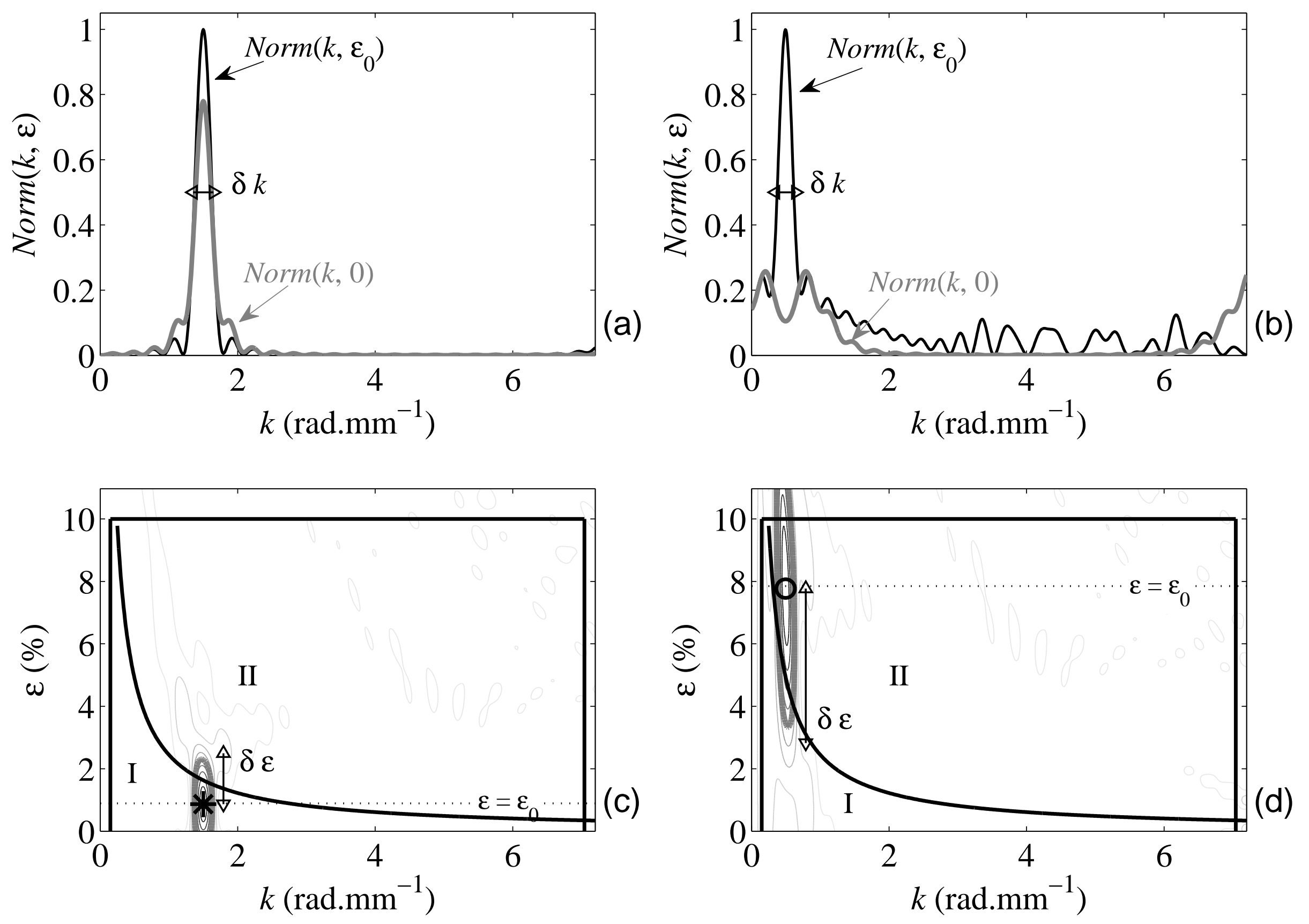

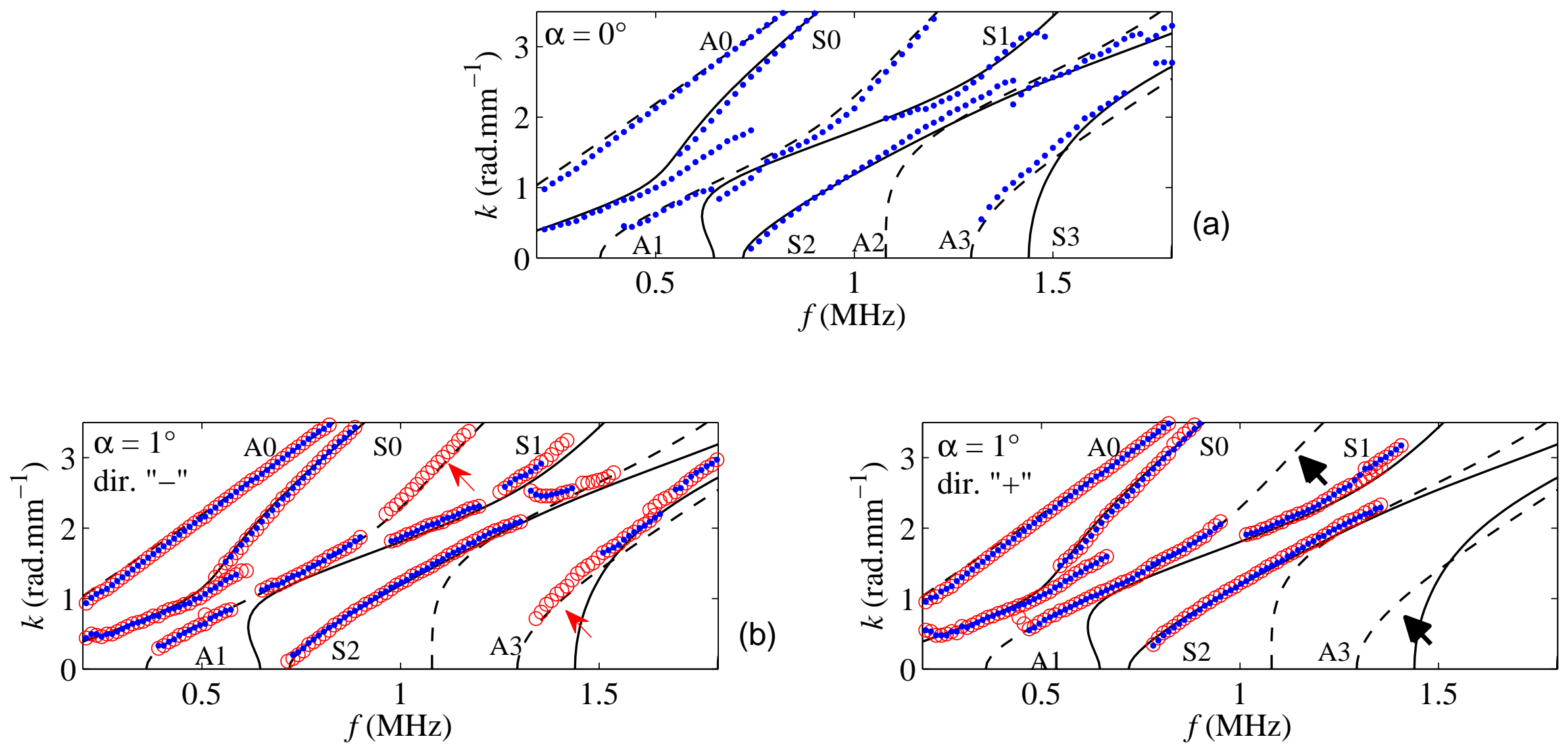

(c)
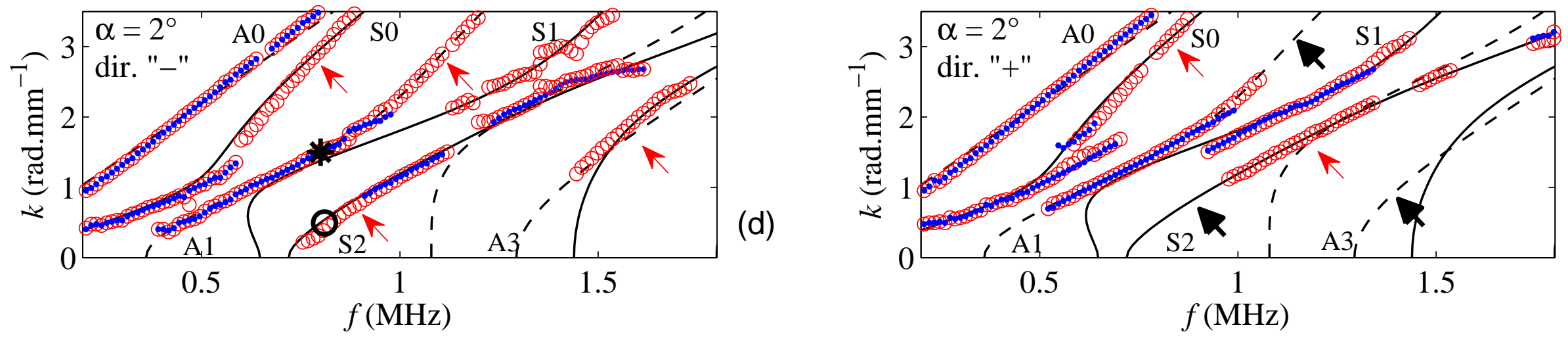

(e) 

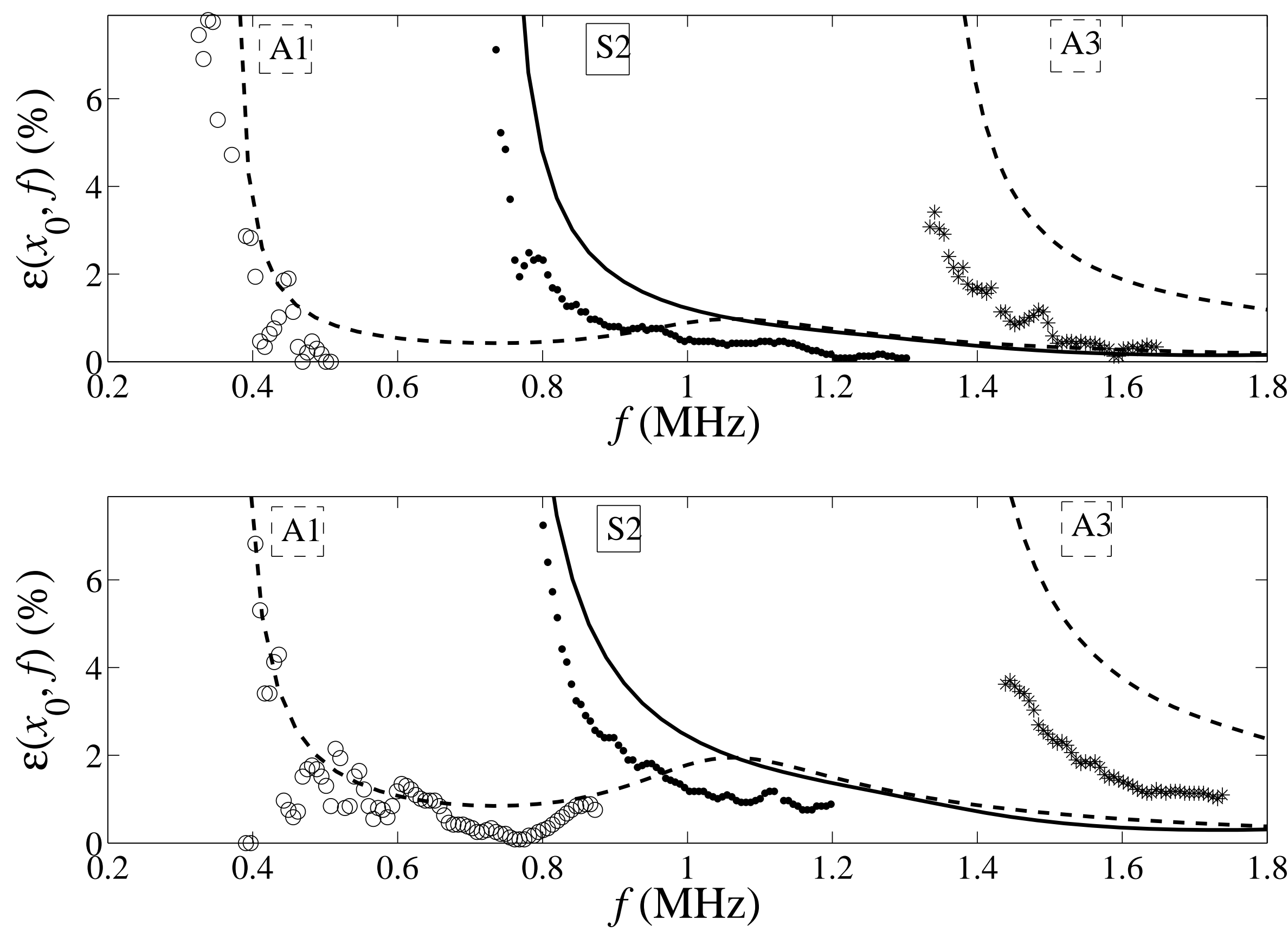